\title{
Substance Use Reported Name
}

National Cancer Institute

\section{Source}

National Cancer Institute. Substance Use Reported Name. NCI Thesaurus. Code C83092.

The literal identifier of the substance in use. 